\title{
On Be star candidates and possible blue pre-main sequence objects in the Small Magellanic Cloud ${ }^{\star}$
}

\author{
R. E. Mennickent ${ }^{1}$, G. Pietrzyński ${ }^{1}{ }^{1,2}$, W. Gieren ${ }^{1}$, and O. Szewczyk ${ }^{2}$ \\ ${ }^{1}$ Universidad de Concepción, Departamento de Física, Casilla 160-C, Concepción, Chile \\ e-mail: pietrzyn@hubble.cfm.udec.cl, wgieren@coma.cfm.udec.cl \\ 2 Warsaw University Observatory, Al. Ujazdowskie 4,00-478, Warsaw, Poland \\ e-mail: szewczyk@astrouw.edu.pl
}

Received 24 April 2002 / Accepted 17 June 2002

\begin{abstract}
Recently the OGLE experiment has provided accurate light curves and colours for about 2 millions stars in the Small Magellanic Cloud. We have examined this database for its content of Be stars, applying some selection criteria, and we have found a sample of $\sim 1000$ candidates. Some of these stars show beautiful light curves with amazing variations never observed in any Galactic variable. We find outbursts in $13 \%$ of the sample (type-1 stars), high and low states in 15\%, periodic variations in $7 \%$, and the usual variations seen in Galactic Be stars in $65 \%$ of the cases. The Galactic counterparts of type- 1 objects could be the outbursting Be stars found by Hubert \& Floquet (1998) after the analysis of Hipparcos photometry. We discuss the possibility that type-1 stars could correspond to Be stars with accreting white dwarf companions or alternatively, blue pre-main sequence stars surrounded by thermally unstable accretion disks. We provide coordinates and basic photometric information for these stars and some examples of light curves.
\end{abstract}

Key words. stars: pre-main sequence - stars: emission-line, Be - stars: variable: general - X-rays: binaries galaxies: individual: SMC

\section{Introduction}

About $1 / 6-1 / 3$ of all Galactic B-type stars with luminosity class III-V once showed $\mathrm{H} \alpha$ in emission and have therefore been classified as Be stars (e.g. Zorec \& Briot 1997). The optical and near-infrared spectra of Galactic Be stars usually display hydrogen, He I and singly ionized metallic emission lines, whose double-peaked shape indicate their origin in a circumstellar gaseous quasi-Keplerian disk. Detailed studies of Be stars in the Small Magellanic Cloud (SMC) have been performed only in recent years, being especially confined to open clusters like NGC 330 (e.g. Keller et al. 1999). These studies show the importance of studying Be stars in the low metallicity environment of the SMC since they serve as probes to test for the mechanisms of disk formation and of global disk oscillations (Baade et al. 2002; Hummel et al. 2001).

Over the past years, the microlensing projects (OGLE, MACHO, EROS) have monitored millions of stars in the Magellanic Clouds and Galactic bulge for variability. The huge resulting photometric databases are very well suited not only for microlensing studies but also for many other issues

Send offprint requests to: R. E. Mennickent, e-mail: rmennick@stars.cfm.udec.cl

$\star$ The complete Tables 1, 3, 5 and 6 are available in electronic form at http://www.edpsciences.org of modern astrophysics, including the distance scale, variable stars, star clusters etc. In particular, the OGLE II project (Udalski et al. 1997), has provided accurate BVI measurements for about 6.5 million stars from the central parts of the Magellanic Clouds (Udalski et al. 1998, 2000). Based on this same material, a unique catalog containing about 68000 variable stars has just been released (Żebruń et al. 2001b).

The aim of this paper is to search the OGLE II database for Be star candidates and provide a much larger sample of these stars in the SMC than previously known. This will provide the basis for posterior follow-up spectroscopic studies of these objects, necessary to fully understand their nature. We will also call attention to several apparently new classes of light curves which appeared during our search for Be stars in the SMC.

The paper has the following structure. Observational data and the applied selection criteria are described in Sect. 2. Results are presented in Sect. 3, and discussed in Sect. 4. In Sect. 5, we present our current conclusions on the underlying nature of the observed types of variability.

\section{The data}

During four years (1997-2000), about 4.5 and 2 million stars were monitored in the LMC and SMC, respectively, in the course of the OGLE II project (Udalski et al. 1997). Typically, 
about $400 I$ and $30 B$ and $V$ observations were secured for each star. An application of a newly developed photometric pipeline (Woźniak 2000; Żebrun et al. 2001a) to the data, based on the DIA method introduced by Alard \& Lupton (1998), resulted in the detection of about 68000 variable stars (Żebruń et al. 2001b). This catalog, apart from presenting positions and light curves for all its objects, provides in addition cross identifications with objects detected during earlier reductions carried out with Dophot. It should be stressed that the positions of the variable stars obtained with the DIA package were obtained from the variable signal only, and may be in principle quite different, especially in the very crowded regions, from the positions obtained from the Dophot photometry. However, the great majority of variable sources detected with DIA were unambiguously cross-correlated with objects detected with Dophot (Żebruń et al. 2001a).

To carry out our search, we inspected the OGLE II SMC BVI maps, and looked for stars matching the usual luminosities and colours observed in Galactic Be stars. Absolute magnitudes of Be stars based on Hipparcos parallaxes (Wegner 2000) indicate that Be stars of luminosity classes IV-V and III have absolute $V$-band magnitudes between -4 and 0 . However, Garmany \& Humphreys (1985) report on a population of extremely luminous non-supergiant Be stars in the Magellanic Clouds with absolute $V$-band magnitudes up to -6 . Therefore, we searched for stars having absolute $V$-band magnitudes between -6 and 0 . Assuming a distance modulus for the SMC of $19.0 \pm 0.2 \mathrm{mag}$, a visual extinction of $0.3 \mathrm{mag}$ and a typical amplitude of the photometric variations of Be stars of 0.2 mag, the above absolute magnitudes imply apparent $V$-band magnitudes between 13.1 and 19.7. In addition to this magnitude constraint, we required that the selected stars should have colours in the range in which Galactic Be stars are usually found, viz. $-0.4<B-V<0.6$ and $-0.35<V-I<0.8$. Using these criteria, we obtained a first sample of $5168 \mathrm{Be}$ star candidates in our database. As a next step, we extracted the I-band light curves for each of these objects from the OGLE II catalog of variable stars (Żebruń et al. 2001b). The light curves were visually inspected and spurious variables, Cepheids and eclipsing binaries rejected. We then proceeded in classifying the remaining 1056 stars according to the appearance of their light curves. Our set of Be star candidates is likely to be complete for stars showing a rms larger than 0.02 mag in the $I$ band. However, we could have missed Be stars either not showing long-term variations, or with $I$-band amplitudes less than $0.02 \mathrm{mag}$.

\section{Results}

Surprisingly, we found not only a large sample of 685 objects showing light curves similar to those observed for Galactic Be stars (we call them type-4 stars, with stochastic or sometimes quasi-periodic variations), but also 139 objects showing outbursts (called type-1 stars), 154 showing high and low states (called type-2 stars), and 78 stars showing periodic or near-periodic long-term oscillations (type-3 stars). 18 type1 stars also showed sudden luminosity jumps like those observed in type- 2 stars. We called them type-1/type- 2 stars. In Figs. 1-6, we show examples of light curves for objects of the
Table 1. Type- 1 stars. $\Delta \Phi$ is the distance (in arcsec) to the nearest star detected with Dophot.

\begin{tabular}{cccccc}
\hline \hline Star & $\Delta \Phi$ & $V$ & $B-V$ & $V-I$ & $\mathrm{rms}$ \\
\hline $003623.36-733922.3$ & 0.601 & 16.158 & -0.233 & -0.212 & 0.012 \\
$003832.64-732234.9$ & 0.076 & 15.264 & -0.139 & -0.117 & 0.01 \\
$003918.20-733656.6$ & 0.245 & 14.548 & 0.484 & 0.714 & 0.017 \\
$003922.09-732531.6$ & 0.031 & 16.931 & -0.041 & -0.146 & 0.019 \\
$004036.15-732921.6$ & 0.04 & 16.548 & 0.13 & -0.03 & 0.062 \\
$004207.86-734501.9$ & 0.136 & 16.804 & -0.055 & 0.049 & 0.02 \\
$004215.08-731710.4$ & 0.097 & 15.966 & -0.157 & -0.055 & 0.019 \\
$004231.84-732200.9$ & 0.024 & 15.376 & -0.106 & -0.019 & 0.077 \\
$004326.72-725910.3$ & 0.439 & 16.858 & -0.128 & -0.127 & 0.018 \\
$004502.43-732318.6$ & 0.137 & 16.777 & -0.048 & 0.039 & 0.012 \\
$004624.69-724657.3$ & 0.609 & 19.333 & 0.203 & 0.463 & 0.045 \\
$004624.74-731941.7$ & 0.102 & 15.851 & 0.069 & 0.169 & 0.09 \\
$004631.41-730335.6$ & 0.085 & 17.113 & -0.118 & -0.002 & 0.04 \\
$004646.81-731849.2$ & 0.054 & 16.701 & -0.013 & 0.207 & 0.015 \\
$004650.39-731017.7$ & 0.092 & 15.513 & -0.105 & -0.022 & 0.014 \\
$004653.17-732330.2$ & 0.327 & 15.252 & -0.033 & 0.012 & 0.014 \\
$004717.43-725227.6$ & 0.119 & 17.071 & 0.353 & 0.403 & 0.136 \\
$004757.42-731050.8$ & 0.122 & 15.714 & -0.036 & 0.041 & 0.02 \\
$004800.14-730728.6$ & 0.055 & 16.354 & -0.226 & 0.046 & 0.101 \\
$004803.08-725404.2$ & 0.347 & 16.704 & -0.127 & -0.126 & 0.015 \\
\hline
\end{tabular}

type-1 to type-4 subclasses. Basic information (OGLE name, distance (in $\operatorname{arcsec}$ ) to the nearest star detected with Dophot, $V$ magnitude, $B-V$ and $V-I$ colours, scatter of $I$-band magnitudes, and optional comments) on these stars is given in Tables 1-6. It is striking that type-2 and type-3 light curves have not been observed in any Galactic variable, to the best of our knowledge, and very few cases of Galactic type-1 stars seems to have been detected (see Sect. 3.1). Therefore all these stars clearly deserve further study. In Figs. 7 and 8 we show the $V$ versus $B-V$, and $V-I$ versus $B-V$ diagrams for the entire sample of stars. It is observed that the number of Be star candidates declines towards the extremes in both diagrams, indicating that the ranges of magnitude and colour we chose for our search were probably near-optimum to include all the Be stars in the database. Regarding the main sequence, most stars are displaced to the red in the $V$ versus $B-V$ diagram; this is in principle consistent with hydrogen recombination (free-bound and free-free) emission from circumstellar envelopes around Be stars (Dachs et al. 1988). Keeping in mind that our data have not been corrected for interstellar or circumstellar reddening, the sharp blue cut-off in the $B-V$ colour is notable. This observational fact is consistent with the vanishing of the Be phenomenon in spectral types earlier than B0, partly due to the efficiency of radiation pressure to remove circumstellar gas in hotter stars.

\subsection{Type-1 stars}

The outbursts shown by type- 1 stars were basically of two types: those characterized by a sudden rise of luminosity followed by a gradual decline, generally lasting tens of days with amplitudes $\lesssim 0.2 \mathrm{mag}$, and those with usually slower declines, lasting hundreds of days with amplitudes $>0.2 \mathrm{mag}$, and 
Table 2. Type-1 stars showing transient quasi-periodic outbursts. ART means "after removing trend", BHS "before high state" and BF "before fading".

\begin{tabular}{ccccccc}
\hline \hline Star & $\Delta \Phi$ & $V$ & $B-V$ & $V-I$ & rms & Time-scale (days) \\
\hline $004957.09-730204.5$ & 1.776 & 17.129 & -0.133 & 0.122 & 0.049 & 332, ART \\
$005039.05-725751.4$ & 0.028 & 17.211 & 0.15 & 0.301 & 0.078 & 136 \\
$005118.72-732846.3$ & 0.158 & 15.765 & 0.039 & 0.248 & 0.013 & 216, ART \\
$005149.34-724134.3$ & 0.069 & 16.026 & -0.013 & 0.163 & 0.02 & 38, BF \\
$005157.18-730811.8$ & 0.183 & 16.544 & -0.058 & 0.092 & 0.025 & 22, ART \\
$005235.60-723751.7$ & 0.03 & 14.573 & -0.145 & -0.114 & 0.045 & 94 \\
$005312.58-725533.6$ & 0.039 & 16.024 & -0.121 & -0.029 & 0.037 & 58 \\
$005321.08-724548.3$ & 0.095 & 15.526 & -0.201 & -0.067 & 0.049 & 231 \\
$005355.66-724359.2$ & 0.151 & 15.56 & -0.092 & -0.057 & 0.114 & 85 or 42, ART, BHS \\
$005623.53-723926.3$ & 0.071 & 16.225 & 0.065 & 0.309 & 0.022 & 215, ART \\
$005758.52-722228.7$ & 0.084 & 15.794 & -0.088 & 0.002 & 0.03 & 40, ART, BF \\
$005802.33-724137.5$ & 0.031 & 17.092 & -0.167 & -0.154 & 0.057 & 16, ART \\
$005950.21-722817.4$ & 0.123 & 17.342 & -0.112 & 0.03 & 0.017 & 173, ART \\
$010001.47-724046.9$ & 0.062 & 15.876 & -0.187 & -0.039 & 0.154 & 133 or 101, ART \\
$010023.52-723302.7$ & 0.025 & 15.6 & -0.194 & -0.079 & 0.022 & 168, ART \\
$010120.64-721118.7$ & 0.097 & 15.489 & -0.065 & 0.078 & 0.019 & 75, ART \\
$010332.59-720326.5$ & 0.086 & 14.91 & -0.207 & -0.129 & 0.146 & 127, ART \\
$010409.79-723835.6$ & 0.086 & 15.325 & -0.097 & 0.046 & 0.026 & 112, BHS \\
$010838.56-723633.5$ & 0.036 & 16.115 & -0.075 & 0.092 & 0.035 & 136 \\
\hline
\end{tabular}

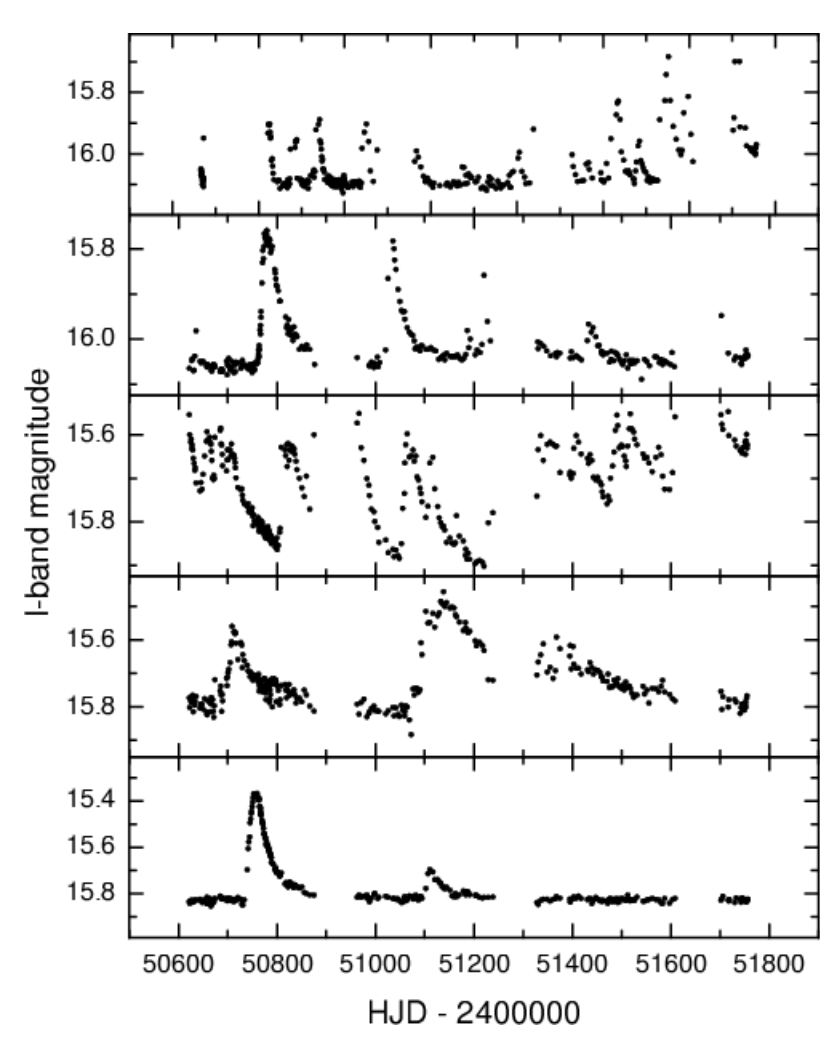

Fig. 1. Examples of type-1 Be star candidates showing sharp outbursts.

characterized by more symmetric rising and fading timescales. In general, in both kinds of outbursts, the stars were redder when brighter and we could not find any evidence of a correlation of outburst rising delay with wavelength. Some stars

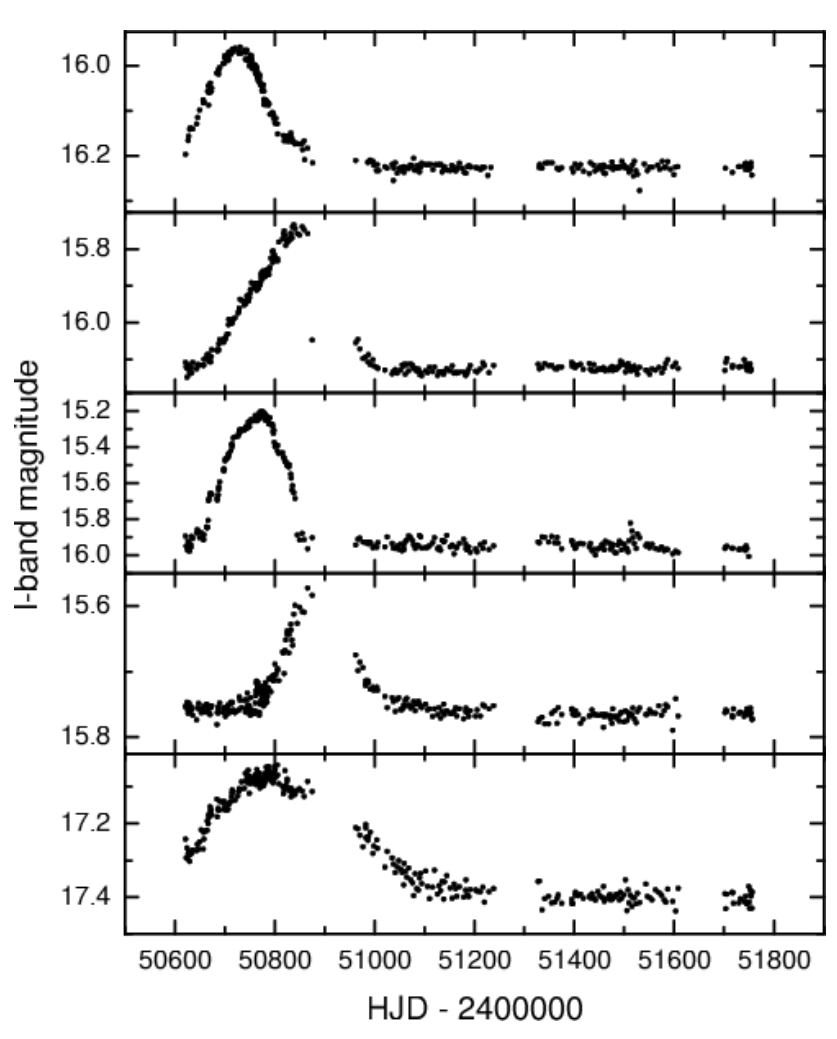

Fig. 2. Examples of type-1 Be star candidates showing hump-like outbursts.

showed both kinds of outbursts, and sometimes it was difficult to classify them when the outburst was faint and not well resolved, or in the cases of noisy light curves. For this reason we do not provide a sub-classification for type- 1 stars. Not always 
these stars are showing a constant quiescence brightness level. For $16 \%$ of type- 1 stars ( 22 objects) it was possible to identify an outburst time-scale, i.e. a typical time for outburst recurrence, although this recursion sometimes was observed only as a transient phenomenon and not during the whole observing period. Basic information for type-1 stars is given in Tables 1 and 2. Exemplary light curves of this type of variable stars are shown in Figs. 1 and 2. The outburst time-scales, given in Table 2, run from 16 to 332 days, with a mean of 126 days. The time-scale histogram shows a decrease of cases toward longer periods; $68 \%$ of the objects show time-scales shorter than 150 days. There is no clear correlation between outburst time-scale, and stellar colour or apparent magnitude.

Our type-1 objects probably correspond to the bumpers found in the Large Magellanic Cloud by Cook et al. (1995) after analysing the MACHO collaboration database. These authors report on the discovery of a group of bright main sequence stars with $V \sim 15-17$ which have a constant luminosity for extended periods of time, but show occasional outbursts at $10-30 \%$ amplitude level. They found that the episodes in more than $50 \%$ of the cases are asymmetric and shorter than about 50 days with a more rapid brightening than dimming. The fact that $\mathrm{H} \alpha$ and $\mathrm{H} \beta$ emission was observed in the spectra of 7 of these stars led Cook et al. (1995) to suggest that these stars could be related to, or maybe identical to, Galactic Be stars.

The Galactic counterparts of type- 1 objects could be the outbursting Be stars found by Hubert \& Floquet (1998) analyzing Hipparcos photometry. They find short-lived outbursts in $\omega \mathrm{CMa}$ and other $13 \mathrm{Be}$ stars, and also a long-lived outburst in $v$ Cyg and another 7 Be stars. The outbursts were preferentially detected in early Be stars with rather low to moderate projected rotational velocities. The average time scale for the recurrence of short-lived outburst is, however, slightly larger than that observed for our SMC sample. Hubert \& Floquet (1998) argue that both kinds of outbursts probably have the same origin and can be explained by discrete high density emitting plasma events seen under low inclination angles with respect to the rotational axis. The number of cases presented by these authors is too small and the photometry too scarce to make a detailed comparison with our type-1 stars, however.

Although at present we cannot exclude the Be star nature of type- 1 objects, we will present, in the next section, two possible alternative explanations for these objects.

\subsection{Type-2 stars and type-1/type-2 stars}

Type-2 stars show sudden brightness jumps of an amplitude of a few tenths of magnitude, with the star remaining at a rather constant brightness level for hundreds of days. Type2 stars usually don't show additional variability, like the irregular variations observed in type-4 stars, except for rather smooth long-term changes. Type- 2 stars can be found in basically the same colour range as type- 1 stars, but type- 2 stars are statistically bluer than type- 1 stars. Eighteen type- 1 stars in our database also showed high-low states like those observed in type-2 stars. These type-1/type- 2 stars were only found in the range of $15.5<V<17$ and in a narrow colour box characterized by $-0.2<B-V<0.2$ and $-0.2<V-I<0.25$. The existence of these "transition" stars supports the conjecture that type- 1 and type- 2 stars could correspond to the same kind of object. Basic information for type- 2 and type-1/type- 2 stars is given in Tables 3 and 4 .

\subsection{Type-3 stars}

In these periodic objects, we searched for periods longer than 0.2 days using a variety of period searching algorithms implemented in graphic C-routines written by us, and also in IRAF. We found periodicities in the range of 17 to 720 days. The accuracy for periods longer than 200 days is low, and these variations can easily be quasi-periodic rather than strictly periodic. $51 \%$ of the periods were found in the range of 17-100 days. The mean period was 135 days. Many of the stars showed significant aliases in the periodogram in addition to the main period, and 2 showed evidence of a variable period. Five stars turned out to be eclipsing binaries, with evidence of additional variability not related to changing aspects of the binary system. In general, type-3 stars were redder when brighter. All the relevant information is given in Table 5. The 9 type- 3 stars in our sample with periods longer than 200 days have $V-I$ colours in the range of $0.0-0.2 \mathrm{mag}$, and $B-V$ colours in the range of $-0.1-0.0$ mag. They correspond to stars with visual magnitudes between 15.5 and 18.0 mag. In general, type-3 stars show similar colours than the other types of stars we are discussing, but they appear more homogeneously distributed in Figs. 7 and 8, with a tendency to concentrate in the red part of the colour-colour diagram.

\subsection{Type-4 stars}

They are the most numerous in our sample, sharing colour ranges with the other types of variables. They usually show stochastic variability in time scales running from days to years (Fig. 6). In some occasions we observed quasi-periodic oscillations on time scales of weeks, and in others short-duration, eclipse-like events. Information on type-4 stars is given in Table 6.

\section{Discussion}

We have reviewed the literature in order to find out if our stars have already been detected as variable stars by other surveys. In the original sample of 5168 objects, we found 8 stars classified as Be stars by Keller et al. (1999). We classified four of them as type-4 (ngc 330/219, ngc 330/235, ngc 346/259 and ngc 346/377), and the others were missed by our visual inspection search method since they show $I$-band rms lower than 0.02 mag (ngc 330/238, ngc 330/1054, ngc 330/1064 and ngc 330/1239). We also found one type-4 star listed as extreme Be-star by Garmany \& Humphreys (1985, their star AV433). Another two type-4 stars are listed as Be/X-ray binaries and one as possible Be/X-ray binary in the compilation by Haberl \& Sasaki (2000, their stars 20, 23 and 6, respectively). The fact that eight type-4 stars have been previously classified as 
Table 3. Type-2 stars.

\begin{tabular}{cccccc}
\hline \hline Star & $\Delta \Phi$ & $V$ & $B-V$ & $V-I$ & rms \\
\hline $004234.39-733058.2$ & 0.043 & 15.989 & -0.148 & 0.002 & 0.021 \\
$004258.05-730230.7$ & 0.036 & 16.059 & 0.028 & 0.176 & 0.019 \\
$004319.46-731705.7$ & 0.099 & 16.403 & -0.056 & 0.132 & 0.032 \\
$004330.69-732034.2$ & 0.028 & 16.428 & -0.194 & -0.177 & 0.009 \\
$004414.25-731215.5$ & 0.086 & 17.297 & -0.107 & -0.028 & 0.023 \\
$004443.05-731316.5$ & 0.068 & 16.217 & -0.072 & 0.028 & 0.017 \\
$004504.35-724449.9$ & 0.076 & 17.906 & 0.336 & 0.549 & 0.051 \\
$004510.40-731648.1$ & 0.104 & 15.855 & -0.105 & -0.022 & 0.009 \\
$004539.31-731040.1$ & 0.433 & 17.849 & -0.104 & 0.702 & 0.088 \\
$004546.74-733233.1$ & 0.008 & 16.284 & -0.032 & 0.158 & 0.032 \\
$004556.84-730919.8$ & 0.068 & 17.128 & -0.057 & 0.031 & 0.013 \\
$004601.22-731821.9$ & 0.11 & 16.374 & 0.033 & 0.255 & 0.018 \\
$004610.97-732535.2$ & 0.037 & 15.439 & 0.009 & 0.032 & 0.146 \\
$004633.48-730352.1$ & 0.026 & 15.489 & -0.066 & 0.063 & 0.04 \\
$004639.77-725241.0$ & 0.047 & 15.614 & -0.179 & -0.173 & 0.011 \\
$004651.07-730215.3$ & 0.048 & 15.801 & -0.026 & 0.176 & 0.026 \\
$004655.32-731207.6$ & 0.094 & 16.164 & -0.113 & 0.103 & 0.03 \\
$004714.29-731044.0$ & 0.09 & 17.635 & -0.022 & 0.043 & 0.022 \\
$004714.53-731349.7$ & 0.131 & 15.068 & -0.196 & -0.172 & 0.009 \\
$004719.31-732533.6$ & 0.11 & 16.449 & -0.074 & 0.145 & 0.073 \\
\hline
\end{tabular}

Table 4. Type-1/type-2 stars. The nomenclature is as in Table 2.

\begin{tabular}{ccccccc}
\hline \hline Star & $\Delta \Phi$ & $V$ & $B-V$ & $V-I$ & rms & Time-scale (days) \\
\hline $004402.00-733129.4$ & 0.221 & 15.456 & -0.003 & 0.232 & 0.016 & 284 \\
$004406.65-732938.2$ & 0.034 & 16.285 & -0.029 & 0.114 & 0.02 & \\
$004650.21-732807.3$ & 0.042 & 15.442 & -0.192 & -0.191 & 0.017 & \\
$004738.11-731126.1$ & 0.12 & 15.484 & -0.114 & 0.022 & 0.008 & \\
$004800.73-732253.0$ & 0.137 & 15.842 & -0.048 & 0.144 & 0.018 & \\
$004817.58-725028.8$ & 0.133 & 16.329 & -0.109 & -0.048 & 0.016 & \\
$004848.27-732611.3$ & 0.062 & 15.462 & -0.098 & 0.016 & 0.067 & 39, BF \\
$004858.25-724119.4$ & 0.054 & 16.157 & -0.074 & 0.076 & 0.015 & \\
$005045.50-730112.9$ & 0.071 & 16.398 & -0.057 & 0.038 & 0.019 & \\
$005053.20-731030.8$ & 0.046 & 15.925 & -0.054 & 0.157 & 0.096 & \\
$005105.65-731311.5$ & 0.044 & 15.911 & -0.032 & 0.208 & 0.047 & 52, ART \\
$005112.13-725656.2$ & 0.08 & 16.196 & -0.082 & 0.09 & 0.034 & \\
$005141.43-731129.9$ & 0.051 & 16 & -0.123 & 0.024 & 0.091 & \\
$005147.58-730924.7$ & 0.066 & 15.629 & -0.073 & 0.077 & 0.045 & \\
$005456.92-731200.4$ & 0.056 & 16.344 & -0.156 & -0.143 & 0.01 & \\
$005651.99-724027.1$ & 0.059 & 15.735 & -0.065 & -0.015 & 0.067 & \\
$005854.06-722841.9$ & 0.114 & 17.014 & -0.04 & 0.134 & 0.042 & \\
$010701.71-724754.9$ & 0.036 & 15.601 & -0.152 & -0.053 & 0.021 & \\
\hline
\end{tabular}

Be stars or Be/X-ray binaries suggests that all type-4 stars might be related with Be stars in some way. Their light curve morphology, quite similar to Galactic Be stars, supports this conjecture.

Type-1 light curves are reminiscent of those observed in FU Orionis stars, thought to be caused by accretion events in protostellar disks around young solar-type stars. There is a notable similarity between the shape of the sharp type-1 outbursts with those observed in the extreme classical T Tauri star EX Lupi (Herbig et al. 2001) and the FU Ori star V 1057 Cyg (Bell et al. 1995). However, the amplitude and duration of the outbursts are respectively factors 20 and 1000 smaller in type- 1 stars.
On the other hand, the general appearance of type-2 light curves, i.e., a rapid rise of luminosity followed by a "plateau" of rather constant brightness resemble (except for a scale factor) the initial stages of the outbursts observed in V1515 Cyg and FU Ori (Bell et al. 1995). Type-1 light curves are also quite similar in shape to those observed in cataclysmic variables (CVs) of the dwarf novae type. However, these objects experience in general much larger amplitude outbursts $(\sim 2-$ 7 mags), have a rather constant luminosity at maximum and usually return to the same quiescence level after outburst.

The outbursts observed in CVs and FU Ori stars have been modeled by mass transfer instabilities in accretion disks 
Table 5. Type-3 stars. ART means "after-removing-trend", ChP changing period, LA low amplitude, MA multiple aliases, and VA variable amplitude.

\begin{tabular}{|c|c|c|c|c|c|c|}
\hline Star & $\Delta \Phi$ & $V$ & $B-V$ & $V-I$ & rms & Period (days) \\
\hline $003813.22-734144.7$ & 0.291 & 16.607 & -0.045 & 0.022 & 0.058 & $187 \pm 19$ \\
\hline $003832.82-734404.2$ & 0.385 & 17.447 & 0.448 & 0.609 & 0.106 & $79 \pm 3, \mathrm{MA}$ \\
\hline 003833.35-731510.1 & 0.177 & 16.467 & 0.019 & 0.084 & 0.064 & $164 \pm 3$ \\
\hline 003927.34-733309.6 & 0.322 & 15.855 & -0.158 & 0.057 & 0.055 & 27, ART, MA \\
\hline 003952.15-730057.7 & 0.53 & 18.815 & -0.042 & 0.022 & 0.077 & $107 \pm 6$, double eclipse? \\
\hline 004019.36-730402.9 & 2.172 & 16.207 & -0.033 & 0.146 & 0.059 & $200 \pm 22, \mathrm{VA}$ \\
\hline 004024.94-734416.1 & 1.58 & 16.531 & -0.018 & 0.04 & 0.01 & $\sim 448$ \\
\hline 004211.38-732437.7 & 0.345 & 17.916 & -0.089 & 0.01 & 0.058 & $74 \pm 3$ \\
\hline $004212.21-734120.0$ & 0.017 & 15.921 & -0.197 & -0.138 & 0.039 & $46 \pm 1$, alias 1.01932 \\
\hline 004323.84-733930.1 & 0.636 & 16.341 & -0.19 & -0.145 & 0.016 & $62 \pm 2$, alias 0.98134 \\
\hline 004336.91-732637.7 & 0.097 & 14.178 & 0.181 & 0.331 & 0.031 & $118 \pm 7, \mathrm{MA}$ \\
\hline 004357.18-732019.9 & 0.139 & 16.51 & 0.386 & 0.668 & 0.065 & $29 \pm 1$ \\
\hline 004454.66-732802.9 & 0.183 & 14.817 & 0.286 & 0.576 & 0.03 & $17.37 \pm 0.15, \mathrm{VA}$ \\
\hline 004517.55-732343.6 & 0.044 & 17.513 & 0.306 & 0.222 & 0.094 & $171 \pm 15$ \\
\hline 004541.10-731219.2 & 0.082 & 17.149 & 0.089 & 0.24 & 0.032 & $182 \pm 18$ \\
\hline 004541.80-724932.3 & 0.046 & 17.252 & 0.308 & 0.48 & 0.206 & $85 \pm 3$ \\
\hline $004554.14-731404.3$ & 0.781 & 15.483 & 0.517 & 0.709 & 0.01 & $99 \pm 4, \mathrm{LA}$ \\
\hline 004633.06-731919.1 & 0.129 & 17.15 & -0.012 & 0.181 & 0.021 & $38 \pm 1$, ART \\
\hline $004633.76-731204.3$ & 0.092 & 14.058 & 0.206 & 0.385 & 0.057 & $184 \pm 18$, eclipsing \\
\hline 004652.03-731423.7 & 0.084 & 15.995 & -0.069 & 0.087 & 0.021 & $\sim 567$, other $67 ?$ \\
\hline 004653.24-724300.0 & 0.495 & 19.469 & 0.03 & 0.12 & 0.166 & $66 \pm 3$ \\
\hline $004702.75-730618.3$ & 0.126 & 16.57 & 0.122 & 0.243 & 0.016 & $17.3 \pm 0.15$, ART, eclipsing \\
\hline 004723.53-730347.0 & 0.11 & 16.328 & -0.043 & 0.162 & 0.016 & $101 \pm 2$, ART, eclipsing \\
\hline $004748.20-731906.1$ & 0.073 & 18.355 & 0.194 & 0.392 & 0.079 & $177 \pm 10$ \\
\hline 004750.14-731316.4 & 0.222 & 15.475 & 0.269 & 0.471 & 0.009 & $30.0 \pm 0.3, \mathrm{LA}$ \\
\hline 004816.01-730635.7 & 0.212 & 15.917 & 0.123 & 0.338 & 0.04 & $26.45 \pm 0.20$, strange phase curve, also type- $1 ?$ \\
\hline $004833.67-732955.6$ & 0.221 & 19.403 & 0.129 & 0.417 & 0.14 & $161 \pm 12$ \\
\hline 004843.23-731415.8 & 0.111 & 16.736 & -0.081 & 0.066 & 0.062 & $\sim 357, \mathrm{VA}$ \\
\hline 004849.06-724309.2 & 0.088 & 17.588 & 0.446 & 0.639 & 0.108 & $150 \pm 15$, rather time-scale \\
\hline
\end{tabular}

(Meyer \& Meyer-Hofmeister 1981; Bell et al. 1995). A heating front propagates across the disk after a certain critical density is reached, pushing the disk into a hot state with high mass transfer rate and consequently high accretion luminosity. Rapid outburst rising times can be produced by outbursts starting in the outer disk and propagating inwards, and slower rising times can be produced by outbursts triggered in the inner disk and propagating outwards. We can speculate that the same phenomenon responsible for FU Ori outbursts is also present in more massive young stars, producing light curve patterns like the ones observed in type- 1 and type- 2 stars. In this view, the smaller outburst amplitude and shorter duration of type-1 outburst when compared with those observed in FU Ori stars should correspond to accretion of matter with lower mass transfer rates. Sharp and hump-like outbursts should correspond to outside-in and inside-out outbursts, respectively. Type-2 stars could be explained by outside-in outbursts followed by a sustained period of a high mass-transfer rate. A characteristic of the FU Ori outbursts is their larger amplitude at shorter wavelengths, contrary to what is observed in type- 1 stars. However, dwarf novae are redder at maximum.

In connection with the above ideas, it is interesting that one of our objects classified as type- 1 was identified by Beaulieu et al. (2001) as a possible pre-main sequence star (ESHC2).
In principle, this is in contradiction with the Cook et al. (1995) classification of some of these objects as Be stars. It is possible that the presence of Balmer emission not necessarily implies a Be star classification for the "bumpers" detected in the LMC by Cook and collaborators. In fact, the low ionization metal absorption lines usually found in optical spectra of Herbig Ae/Be stars could be hard to detect in low metallicity stellar photospheres like those found in the SMC and LMC. Therefore, it is reasonable to ask if perhaps all of type- 1 and type- 2 objects are actually pre-main sequence stars with FU Ori-like accretion disk activity. In this case, the mass accretion rates need to be much higher than those observed in normal Be star discs, or even in Be/X-ray binaries, to yield luminosities comparable or larger than the luminosity of the accreting B-type star. Since the nebulosities defining Herbig Ae/Be objects cannot be resolved at the distance of the Magellanic Clouds, the answer to the above question needs to await future spectroscopic studies, especially in the infrared, and possibly very high-resolution imaging studies which could reveal the surrounding nebulosities. We must keep in mind, however, that Hipparcos photometry of Galactic Herbig Ae/Be stars apparently does not reveal type-1 like activity (van den Ancker et al. 1998). Also, the optical light of some Galactic Be stars showing outbursts, like $\lambda$ Eri and $\mu$ Cen, is apparently dominated by the stellar 
Table 6. Type-4 stars.

\begin{tabular}{cccccc}
\hline \hline Star & $\Delta \Phi$ & $V$ & $B-V$ & $V-I$ & rms \\
\hline $003618.14-734624.6$ & 0.086 & 14.929 & 0.353 & 0.779 & 0.279 \\
$003618.15-734247.4$ & 1.419 & 16.177 & -0.131 & $-1 \mathrm{E}-3$ & 0.036 \\
$003619.44-732553.1$ & 0.132 & 19.519 & 0.258 & 0.516 & 0.182 \\
$003621.53-732610.6$ & 0.05 & 14.741 & 0.067 & 0.214 & 0.124 \\
$003623.02-734651.0$ & 0.016 & 19.551 & 0.484 & 0.531 & 0.304 \\
$003645.28-733259.7$ & 1.863 & 15.59 & -0.024 & 0.2 & 0.015 \\
$003652.74-732857.9$ & 3.222 & 14.397 & 0.081 & 0.177 & 0.016 \\
$003715.60-734546.0$ & 1.804 & 16.09 & 0.453 & 0.647 & 0.013 \\
$003726.27-731420.0$ & 0.511 & 19.63 & 0.318 & 0.559 & 0.25 \\
$003730.99-731112.4$ & 1.965 & 15.846 & 0.109 & 0.209 & 0.01 \\
$003744.41-730447.6$ & 0.109 & 16.996 & -0.087 & -0.051 & 0.028 \\
$003804.71-735150.5$ & 0.329 & 19.434 & 0.009 & 0.092 & 0.065 \\
$003810.16-730138.4$ & 1.22 & 17.296 & 0.59 & 0.767 & 0.02 \\
$003813.77-731340.0$ & 0.208 & 17.335 & -0.081 & 0.116 & 0.016 \\
$003817.01-734852.6$ & 0.025 & 15.199 & 0.462 & 0.709 & 0.28 \\
$003827.69-733314.5$ & 1.499 & 16.126 & -0.156 & 0.124 & 0.021 \\
$003833.29-732629.4$ & 1.317 & 16.725 & 0.414 & 0.619 & 0.011 \\
$003836.25-734813.4$ & 0.303 & 15.383 & -0.14 & -0.082 & 0.022 \\
$003837.66-731158.3$ & 0.093 & 14.894 & -0.085 & 0.057 & 0.028 \\
\hline
\end{tabular}

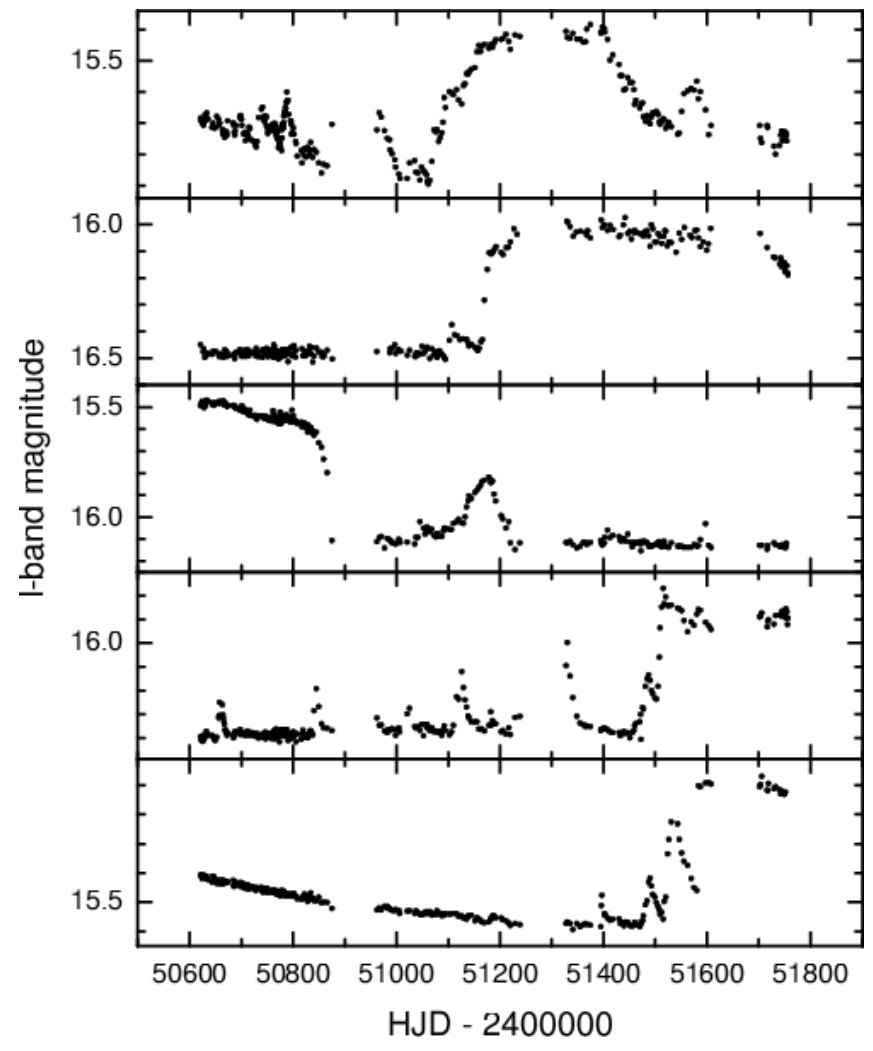

Fig. 3. Examples of type-1/type-2 Be star candidates showing outbursts and high-low states.

continuum during quiescence, and the spectrum does not reveal the strong emission lines typical of massive Herbig Ae/Be envelopes. Although it is not clear if these Galactic Be stars are the same kind of object that type-1/type- 2 stars, we recognize that these two facts are the weak points in the pre-main

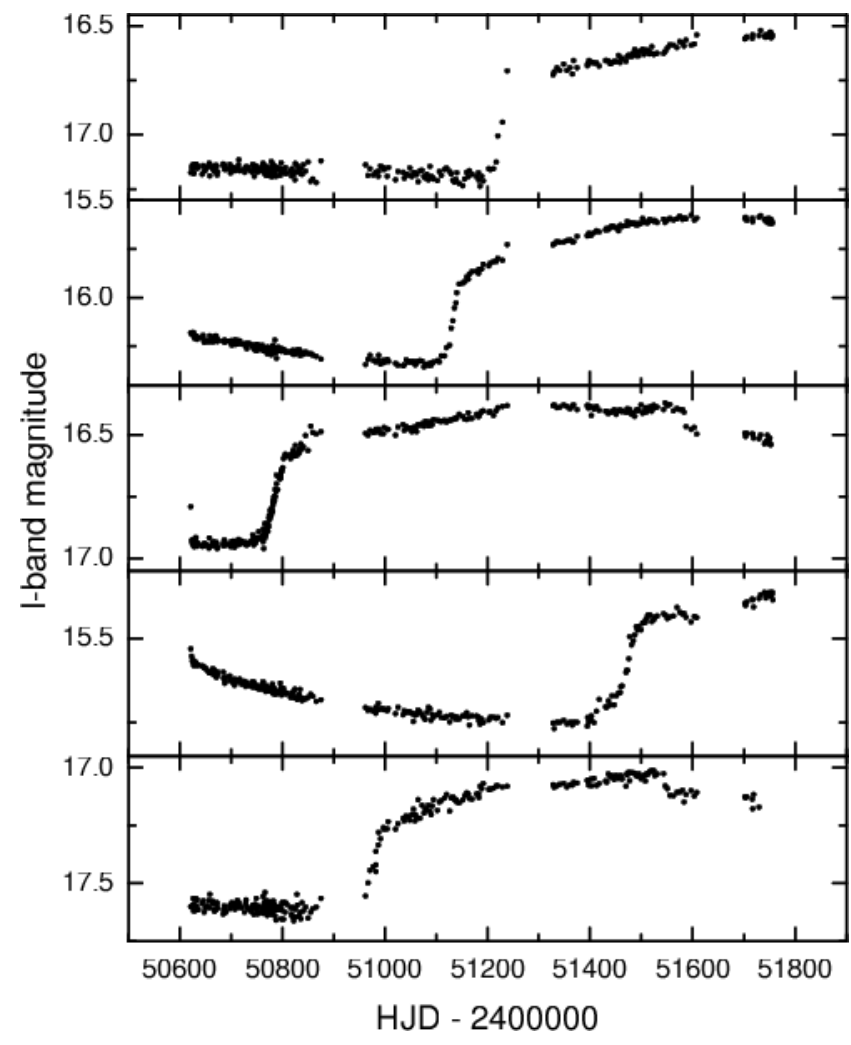

Fig. 4. Examples of type-2 Be star candidates showing high and low states.

sequence scenario. We therefore offer an alternative explanation in the next paragraph.

Type- 1 star outbursts resemble also in shape the optical outbursts observed in some Be/X-ray binaries (e.g. A 0538-66, Densham et al. 1983), except for their much smaller 


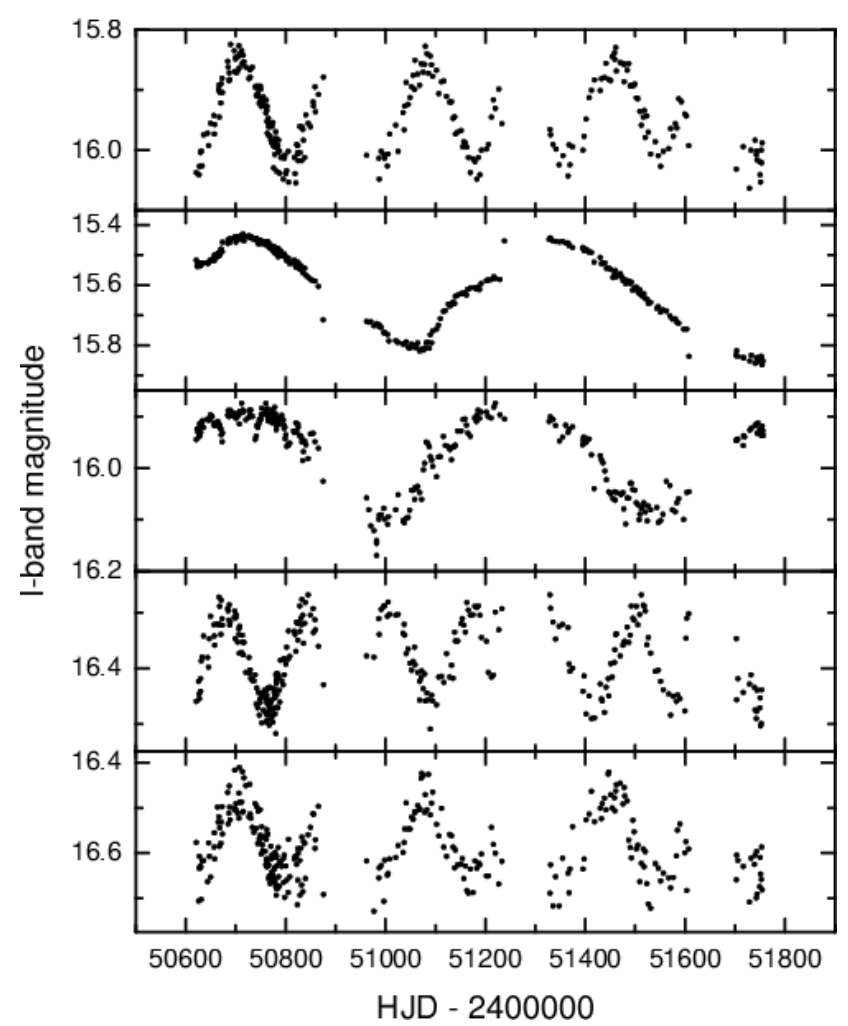

Fig. 5. Examples of type-3 Be star candidates showing periodic oscillations.

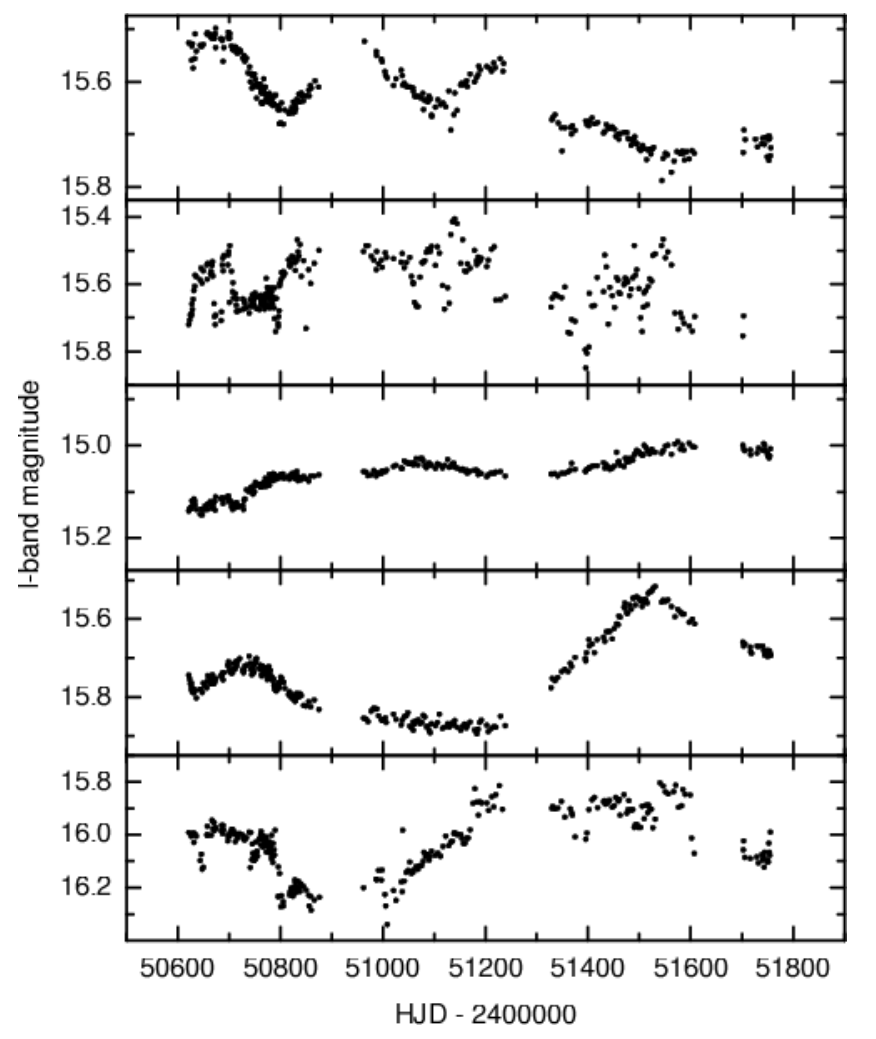

Fig. 6. Examples of type-4 Be star candidates showing random variability. amplitudes and redder colours. It is currently assumed that in these objects a neutron star companion undergoes episodes of mass accretion when passing through the envelope of a Be star primary (e.g., Okazaki \& Negueruela 2001). These high-energy phenomena are no doubt relevant in the X-ray domain, but should not show up at optical and near infrared wavelengths. In addition, the optical counterparts of Galactic and Magellanic Cloud Be/X-ray binaries have spectral types earlier than B2 (Negueruela 1998). This is in principle contrary to the distribution of $B-V$ colours in Fig. 7. However, the similarity between the X-ray type-I and type-II outbursts observed in Be/X-ray binaries and the narrow and hump-like outburst observed in type1 stars suggest that they could be due to accretion by a white dwarf, not a neutron star. The $\mathrm{Be}+\mathrm{WD}$ system should produce less energetic outburst, eventually much prominent in the optical and infrared. Favouring this hypothesis is the fact that many $\mathrm{Be}+\mathrm{WD}$ binaries, even with late-type $\mathrm{B}$ stars, are expected from evolutionary calculations (Raguzova 2000; Waters et al. 1989). Only few candidates for these systems have been found up to now, likely due that the white dwarfs, if present, should be hard to detect (Apparao 1991). The hypothesis of accreting white dwarf companions would naturally explain some of the type-1 light curves. The same model for periodic X-ray outbursts in Be/X-ray binaries by Okazaki \& Negueruela (2001) could eventually be applied to Be+WD systems. In this model, periodic narrow outbursts occur in systems with high orbital eccentricity, every time that the compact object captures gas from the disc at every periastron passage. Hump-like outbursts occurs in systems with low eccentricity, when the outer disc eventually passes beyond the orbit of the companion. Accordingly to Apparao (1991), observations of time delays between enhancements of optical line and continuum emission could identify Be stars with white dwarf companions. We must keep in mind, that only a small fraction of type-1 objects shows periodic or quasiperiodic outbursts, however.

Concerning type-3 stars, they can be ruled out to be Cepheids, since their large periods (sometimes hundreds of days) are incompatible with their inferred luminosities. On the other hand, the periods are one or two orders of magnitude longer than those observed in Galactic pulsating B stars. This hints at the possibility that type- 3 variability is linked to the envelope rather than to the star itself. There is evidence for global one-armed oscillations in the envelopes of Galactic Be stars (Okazaki 1997), but the associated photometric variations are not so strictly periodic as those observed in type3 stars, the time-scales are longer, about 7 years in average, and, from a theoretical point of view, the oscillations are expected to disappear in the low metallicity environment of the SMC or, alternatively, to slow down, yielding periods in excess of several years (Hummel et al. 2001), in disagreement with the observed type- 3 oscillations. The distribution of type3 stars in the colour-colour diagram of Fig. 8 suggests that these stars are perhaps not be linked to the Be star phenomenon at all. Preliminary spectroscopy of one type-3 object seems to corroborate this view. It shows double $\mathrm{H} \alpha$ emission with a deep central absorption and total equivalent width $\sim 100 \AA$, too large for an isolated Be star (Mennickent et al. in preparation). 


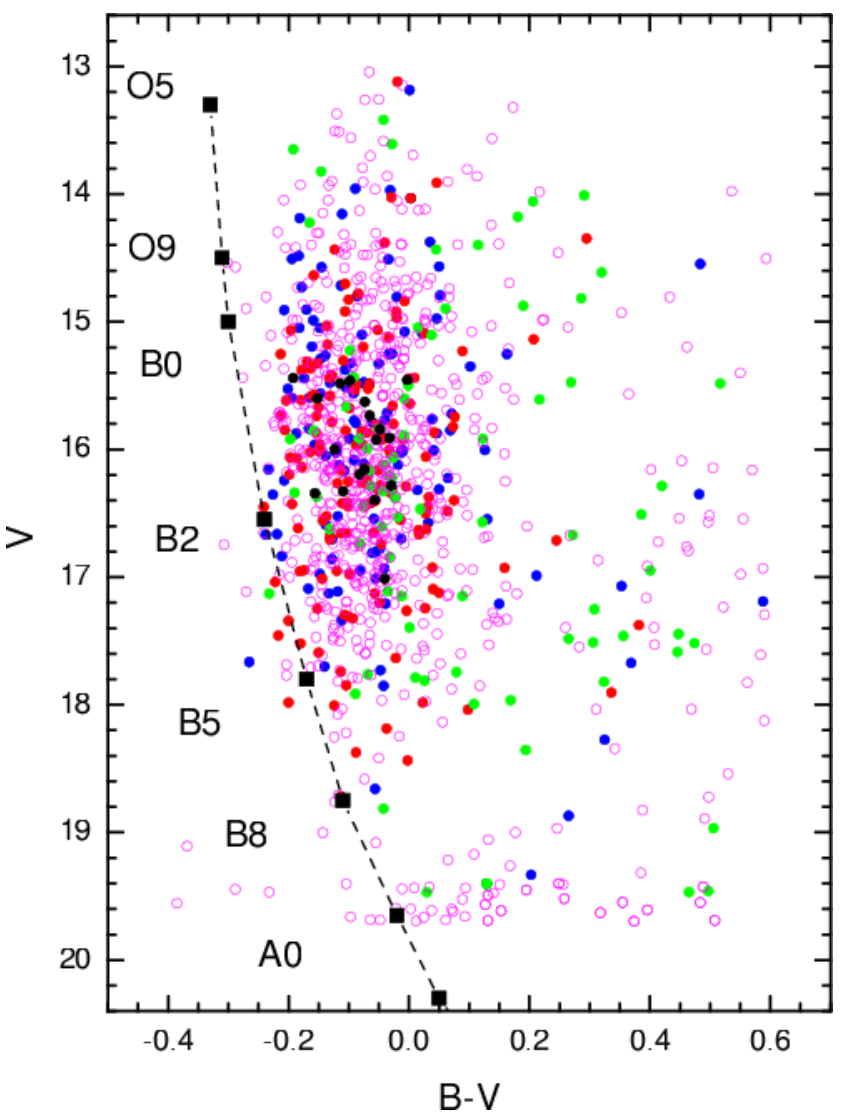

Fig. 7. The $V$ vs. $B-V$ diagram for the sample stars. Blue dots correspond to type-1 stars, red dots to type-2 stars, green dots to type- 3 stars, circles to type- 4 stars and black dots to type- 1 stars showing high and low states, as type- 2 stars. The track of the main sequence (Allen 2000) is shown for reference. Note the sharp cut-off at the blue edge.

We speculate that type- 3 stars could be young objects with massive envelopes ongoing some kind of oscillation.

\section{Conclusions}

We have presented a sample of $\sim 1000$ Be star candidates in the Small Magellanic Cloud, giving positions and basic photometric information along with periodicities when present. Many of these objects show apparently new kinds of photometric variability never seen before, and this feature enabled us to carry out an empirical classification based on the light curve appearance. There is evidence that some of these objects are truly Be stars, but spectroscopy is needed to confirm this suspicion. On the other hand, the hypothesis that at least part of the type1 and type- 2 stars are Be-stars with accreting white dwarfs, or alternatively, pre-main sequence stars showing accretion disk instabilities, should be studied more thoroughly.

The understanding of the phenomena causing the photometric variability of the stars discussed in this paper requires knowledge of the spectral energy distributions of these stars. We plan to discuss, in a forthcoming paper, the detailed photometric properties of these objects along with spectroscopic data. It should be interesting to test the accretion scenario for

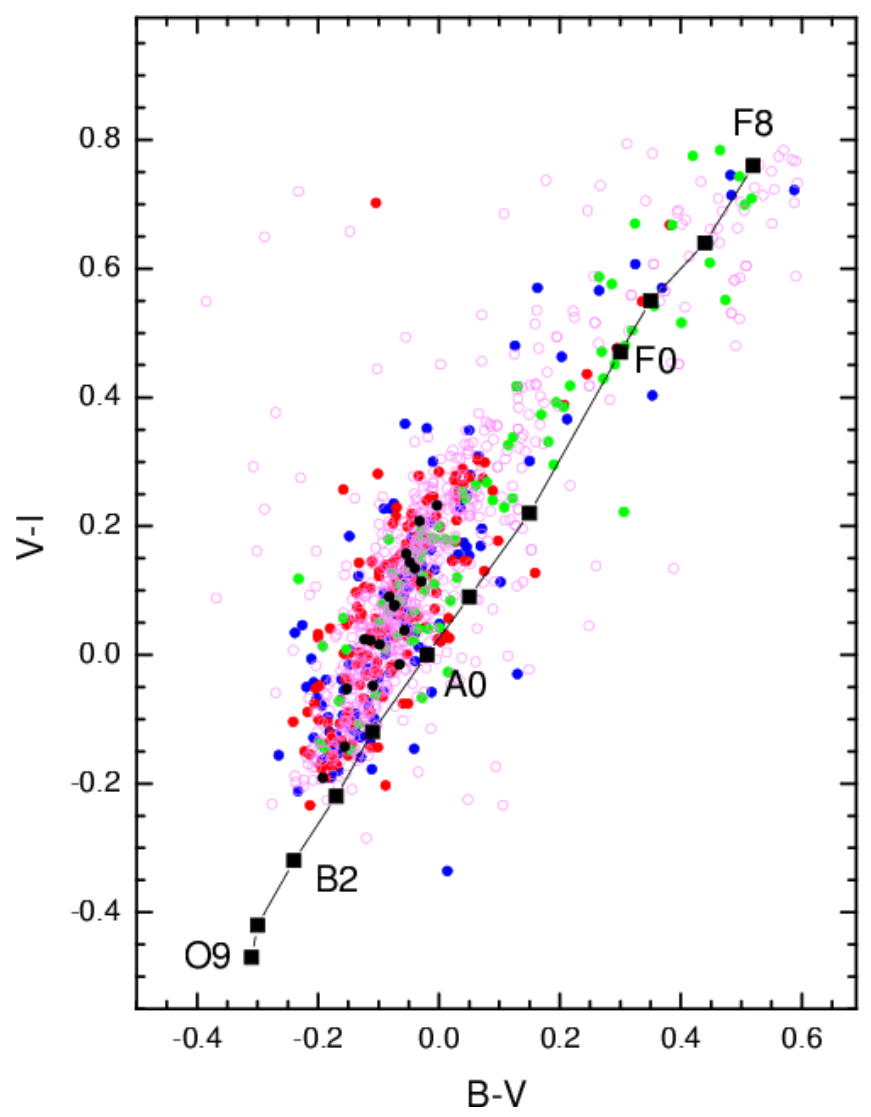

Fig. 8. The $V-I$ vs. $B-V$ diagram for the sample stars. The track of the main sequence (Allen 2000) is shown as a reference. Meaning of the symbols are as in Fig. 7.

type-1 stars. Since the theory of accretion is rather well developed, it could be a source of physical information about the environments surrounding these objects. We also plan to search the OGLE-II database for Be stars in the Large Magellanic Cloud. It should be interesting to investigate if the same types of light curves are observed in the LMC, whose metallicity is intermediate between the SMC and our Galaxy, and how the light curve properties are modified by the change in metallicity.

Acknowledgements. This work was supported by Grant Fondecyt 1000324 and DI UdeC 202.011.030-1.0. The authors are very grateful to Professor Bohdan Paczynski for many interesting suggestions on this paper. We would also like to thank the OGLE collaboration for making their data public domain, thus enabling us to carry out this research. We also acknowledge the anonymous referee who made valuable comments to improve a first version of this manuscript.

\section{References}

Alard, C., \& Lupton, R. H. 1998, ApJ, 503, 325

Allen, C. W. 2000, in Allen's Astrophysical Quantities, fourth edition, ed. A. Cox (AIP Springer-Verlag New York)

Apparao, K. M. V. 1991, A\&A, 248, 139

Baade, D., Rivinius, T., Štefl, S., \& Kaufer, A. 2002, A\&A, 383, L31

Beaulieu, J.-P. Beaulieu, J.-P., de Wit, W. J., Lamers, H. J. G. L. M., et al. 2001, A\&A, 380, 168

Bell, K. R., Lin, D. N., Hartmann, L. W., \& Kenyon, S. J. 1995, ApJ, 444,376 
Cook, K. H., Alcock, C., Allsman, H. A., et al. 1995, in Astrophysical Applications of Stellar Pulsation, ed. R. S. Stobie, \& P. A. Whitelock, ASP Conf. Ser., 83, 221

Dachs, J., Kiehling, R., \& Engels, D. 1988, A\&A, 194, 167

Densham, R. H., Charles, P. A., Menzies, J. W., van der Klis, M., \& van Paradijs, J. 1983, MNRAS, 205, 1117

Garmany, C. D., \& Humphreys, R. M. 1985, AJ, 90, 2009

Haberl, F., \& Sasaki, M. 2000, A\&A, 359, 573

Herbig, G. H., Aspin, C., Gilmore, Alan, C., et al. 2001, PASP, 113, 1547

Hubert, A. M., \& Floquet, M. 1998, A\&A, 335, 565

Hummel, W., Gässler, W., Muschielok, B., et al. 2001, A\&A, 371, 932

Keller, S. C., Wood, P. R., \& Bessell, M. S. 1999, A\&AS, 134, 489

Meyer, F., \& Meyer-Hofmeister, E. 1981, A\&A, 104, L10

Negueruela, I. 1998, A\&A, 338, 505

Okazaki, Y. 1997, A\&A, 318, 548

Okazaki, A. T., \& Negueruela, I. 2001, A\&A, 377, 161

Raguzova, N. V. 2000, in The Be phenomenon in Early-Type Stars, IAU Colloq. 175, ASP Conf. Ser. 214, ed. M. A. Smith, H. F. Henrichs, \& J. Fabregat, 693
Udalski, A., Kubiak, M., \& Szymanski, M. 1997, Acta Astron., 47, 319

Udalski, A., Szymanski, M., Kubiak, M., et al. 1998, Acta Astron., 48, 147

Udalski, A., Szymanski, M., Kubiak, M., et al. 2000, Acta Astron., 50, 307

van den Ancker, M. E., de Winter, D., \& Tjin A Djie, H. R. E. 1998, A\&A, 330, 145

Waters, L. B. F. M., Pols, O. R., Hogeveen, S. J., Cote, J., \& van den Heuvel, E. P. J. 1989, A\&A, 220, L1

Wegner, W. 2000, MNRAS, 319, 771

Williams, G. 1991, AJ, 101, 1929

Wozniak, P. 2000, Acta Astron., 50, 421

Żebruń, K., Soszynski, I., Wozniak, P. R., et al. 2001a, Acta Astron., 51,303

Żebruń, K., Soszynski, I., Wozniak, P. R., et al. 2001b, Acta Astron., 51,317

Zorec, J., \& Briot, D., 1997, A\&A, 318, 443 